\title{
An Introverted Starburst: Gas and SSC Formation in NGC 5253
}

\author{
J. L. Turner \\ Department of Physics and Astronomy, UCLA, Los Angeles, CA 90095 \\ $U S A$ \\ S. C. Beck \\ Department of Physics and Astronomy, Tel Aviv University, Ramat \\ Aviv, Israel
}

\begin{abstract}
High resolution Brackett line spectroscopy with the Keck Telescope reveals relatively narrow recombination lines toward the embedded young super star cluster nebula in NGC 5253. The gas within this nebula is almost certainly gravitationally bound by the massive and compact young star cluster.
\end{abstract}

\section{Introduction}

NGC 5253 is a nearby dwarf galaxy that has created many luminous young star clusters during the past 2-50 Myr (Calzetti et al. 1997, Tremonti et al. 2000). Among these young super star clusters (SSCs) is an extremely young, embedded SSC visible only in the infrared and radio (Beck et al. 1996, Turner et al. 1998, 2003, Gorjian et al. 2001). The radio nebula is unusual in that it is optically thick at $2 \mathrm{~cm}$, indicating that it is a compact HII region, yet it has has a diameter of $1-2$ pc (Turner et al. 1998, 2000). The unusually high volume of relatively dense $\left(\mathrm{n}_{\mathrm{e}} \sim 10^{4}-10^{5} \mathrm{~cm}^{-3}\right)$ gas in the "supernebula" signals the presence of a large number of stars. The cluster is estimated to contain 4000-6000 O stars (Turner et al. 1998, Meier et al. 2002), have a mass of a few hundred thousand solar masses, and a luminosity of $\sim 10^{9} \mathrm{~L}_{\odot}$ (Gorjian et al. 2001), all within a volume of 1-2 pc (Turner et al. 2000).

\section{Brackett Spectroscopy Reveals Narrow Lines in the SSC Nebula}

High resolution Brackett line spectroscopy confirms the radio-inferred properties of the supernebula. Using NIRSPEC on the Keck Telescope with a spectral resolution of $\sim 25,000\left(12 \mathrm{~km} \mathrm{~s}^{-1}\right)$, we detected $\operatorname{Br} \alpha(4.05 \mu \mathrm{m})$ and $\operatorname{Br} \gamma(2.17 \mu \mathrm{m})$ toward the brightest K-band source in NGC 5253. Mapping of the inner $20^{\prime \prime}$ region confirms that this region dominates the Brackett line emission. The Brackett fluxes are in excellent agreement with radio fluxes (Turner et al. 2003).

The unusual aspect of the recombination lines is that they are relatively narrow for the size and luminosity of the nebula. The Brackett lines have widths of $75 \mathrm{~km} \mathrm{~s}^{-1}$, FWHM, consistent with those of the radio recombination lines 
(Mohan et al. 2001). The diameter of the nebula, based on subarcsecond VLA images, is $0.7 \mathrm{pc}$ (Turner et al. 2000). For this size, escape velocity is from 40 $\mathrm{km} \mathrm{s}^{-1}$ for a cluster of only O stars to $120 \mathrm{~km} \mathrm{~s}^{-1}$ for a Salpeter IMF down to M stars, and virial linewidths will be somewhat smaller. So the observed linewidths of the nebula imply that it is actually in or close to gravitational equilibrium. Nebulae in the Galaxy are not confined by gravity, nor is 30 Doradus. This HII region represents a very different dynamical situation.

$\mathrm{HI}$ and CO gas have been detected from NGC 5253. Based on VLA HI images, Kobulnicky \& Skillman (1995) suggested that the HI was rotating about the major axis and that NGC 5253 is perhaps a polar ring galaxy. $\mathrm{CO}$ has been detected along the dust lane in NGC 5253 (Turner, Hurt, \& Beck 1997), and Meier et al. (2002) concluded that the CO kinematics were more consistent with inflow onto the galaxy than rotation. In either case, there is no clear evidence for dispersal of gas from the starburst even though NGC 5253 has soft X-ray emission suggesting the presence of stellar winds (Martin \& Kennicutt 1997).

\section{Conclusions}

NGC 5253 contains an active starburst of luminosity $1 \times 10^{9} \mathrm{~L}_{\odot}$, confined to an area less than 1-2 pc in diameter. The young cluster responsible for this luminosity is embedded, surrounded by a compact, dense nebula. The low linewidth of $75 \mathrm{~km} \mathrm{~s}^{-1}$ seen in the Brackett recombination lines and the very small size of the source suggest that this nebula is gravitationally bound to the embedded cluster. NGC 5253 represents a stage in starburst evolution in which the galaxy is not yet affecting its surroundings: an introverted starburst.

Acknowledgments. This research is supported by NSF Grant 0307950 .

\section{References}

Beck, S. C., Turner, J. L., Ho, P. T. P., Kelly, D. \& Lacy, J. H. 1996, ApJ, 457, 610

Calzetti, D. et al. 1997, AJ, 114, 1834

Gorjian, V. 1996, AJ, 1120, 1886

Kobulnicky, H. A., \& Skillman, E. D. 1995, ApJ, 454, L121

Martin, C. L., \& Kennicutt Jr., R. C. 1995, ApJ, 447, 171

Meier, D. S., Turner, J. L., \& Beck, S. C. 2002, AJ, 124, 877

Mohan, N. R., Ananthamariah, K. R. \& Goss, W. M. 2001, ApJ, 557, 659

Tremonti, C. A., Calzetti, D., Leitherer, C., \& Heckman, T. M. 2001, ApJ, 555, 322

Turner, J. L., Ho, P. T. P., \& Beck, S. C. 1998, AJ, 116, 1212

Turner, J. L., Beck, S. C., \& Ho, P. T. P. 2000, ApJ, 532, L109

Turner, J. L., Beck, S. C., Crosthwaite, L. P., Larkin, J. E., McLean, I. S., \& Meier, D. S. 2003, Nature, 423, 621 